\title{
A Classification of Educational Mobile Use for Learners and Teachers
}

\author{
Oumayma Chergui, Ahlame Begdouri, and Dominique Groux-Leclet
}

\begin{abstract}
In recent years, mobile learning has been a fast growing concept. Through the great number of conducted experiments, many researchers and institutions recognize the potential of mobile technologies as a learning tool. However, a wide adoption of the concept itself is still challenging. In fact, one of the difficulties faced by a teacher is the choice of the appropriate tool and, more generally, the appropriate use of mobile for a specific educational context. In this paper, we propose a generic classification of educational mobile uses based on learning strategies paradigm, and taking into account both learners' and teachers' activities. We validate our findings by some statistics of the most downloaded educational mobile apps from the most popular mobile App Stores.
\end{abstract}

Index Terms-Educational mobile apps, learning strategies, mobile learning, teacher's activity.

\section{INTRODUCTION}

Mobile technologies are gradually increasing and diversifying across every sector of society including education, by opening up exciting new possibilities for learning and teaching; which falls under the "mobile learning" concept. Mobile learning could be implemented in various forms and contexts. Whether it's a complementary activity to e-learning and traditional learning, or a separate informal learning activity.

Although mobile devices are creating new forms of knowledge and new ways of accessing it, which could be very beneficial for learning, the adoption of mobile learning can still be challenging for any teacher or instructional designer wishing to implement a mobile learning solution. There is a lack of documentation and support on the possible ways mobile can be used for learning and teaching. Whether it is decided to use existing mobile applications (apps) or to implement new tools, the instructor needs to make a lot of effort exploring tens of thousands of educational apps available and hundreds of research works to define the possible ways mobile could be used for education, before making the choice of the appropriate tools.

In order to address this issue, we investigated how best mobile tools could be used for educational purposes to support every elementary action of the whole learning process. Our purpose is to propose a generic classification of educational mobile use that takes into consideration learners'

Manuscript received January 25, 2016; revised March 15, 2016.

O. Chergui and A. Begdouri are with the SIA laboratory, FST, University of Sidi Mohamed Ben Abdellah, Fez, Morocco (e-mail: oumayma.chergui@usmba.ac.ma, abegdouri@gmail.com).

D. Groux-Leclet is with the MIS laboratory, University of Picardie Jules-Verne, Amiens, France (dominique.groux@u-picardie.fr). and teachers' point of view. These use categories should be independent from the technological changes that may occur in the near future. They should be also suitable for several learning forms: university, primary and secondary schools as well as informal and life-long learning.

In this paper, we start by presenting some current cutting-edge mobile technologies, then we present some experiments of adopting m-learning from previous research works and a synthesis of previous classifications related to $\mathrm{m}$-learning. At a second stage, we introduce our classification criteria, describe the different dimensions of this classification and argue with some statistics from a study that we have conducted on the most popular mobile app Stores.

\section{General ConteXt}

\section{A. Mobile as a Technology}

Since the early 2000s, Mobile learning has been mainly possible thanks to technological advances in both mobile devices and wireless networks. The first generation of mobile devices presented many limitations [1], in particular small low-resolution screens, poor audio quality, awkward text entry, limited memory and slow Internet connectivity. These technical limitations didn't allow much creativity in using mobile technology for learning purposes [2]-[4].

Nowadays we have overcome this obstacle. Current mobile technology expands the ability to access, store, create, and modify information. Smartphones and tablets have considerably large screens with high resolution and clarity, more powerful processors, large storage capacity, longer battery life etc. Some of their functionalities even surpass in some ways what is available on computers, as many include GPS chips, accelerometers, compasses, high-resolution cameras, proximity sensors etc. Also, network standards and technologies are getting more powerful with higher-performance (e.g. 5G, Wifi-Gigabit, LTE-Advanced, etc.) which makes it possible to transmit large amounts of data. Having such powerful devices available anytime, anyplace provides tremendous opportunities for educational use.

\section{B. Mobile as a Learning Tool}

Mobile learning, as defined by O'Malley et al. [5], is any sort of learning that happens when the learner is not at a fixed, predetermined location, or learning that happens when the learner takes advantage of the learning opportunities offered by mobile technologies. Some advocates of mobile learning attempt to define and conceptualize it in terms of devices and technologies; other advocates define and conceptualize it in terms of learners mobility and learning mobility [6].Whatever the perspective of viewing mobile learning, most authors 
agree that mobile technology can provide particular benefits for learners, mainly its portability, interactivity, ease of use, high efficiency, flexibility and reliability [7].

In the formal context, as an institutional initiative or teachers' personal initiative, many educational organizations have integrated mobile technologies with their conventional learning activities in order to create better learning opportunities for their students. Through the study of these experiences, we have identified three main ways through which these technologies can be used:

- Mobile access to existing e-learning platforms (LMSs),

- Use of mobile apps available in the existing app stores.

- Design and implementation of new mobile apps according to specific learning needs.

In the first category, mobile learning is considered as an extension of e-learning. Therefore, mobile access was either achieved: 1) through a simple user interface to already existing functionalities of an e-learning platform. This is the case in [8] where students used their mobile devices to access the chat and forums tools of the university LMS (Interact 2, based on Blackboard) in order to interact with peers, 2) by adding to the LMS some features and functionalities specifically adapted to the mobile context. For instance, in [9], a Moodle plug in (MLE-Moodle) has been developed including a learning part (flash card trainer, notices and messages) and an evaluation part (quizzes). The plug in does not allow viewing the content available on the desktop version but has been designed as a new functionality adapted to mobile context; Or 3) by doing both, which is the case of the Blackboard LMS [10], it provides several mobile tools allowing access to its web based core (important activities' stream, teacher's assignments, projects progress, chat, etc.) and other tools specifically designed for mobile context (events notifications, real-time SMS surveys, etc.).

In the second category, and independently of any LMS, teachers made benefit from the large number of mobile apps available for download in the app stores. An app is chosen to help achieving specific learning objectives among students. For example, in [11], students received, throughout the semester, a list of apps from their French and German language teachers. They engaged in various in-class tasks using these apps such as exploring newspaper headlines, dictionary and grammar apps, listening to music and watching Youtube videos in the target language, generating thereby, a rich basis for discussions in class. Students were also required to do homework assignments using their mobile devices: preparing video interviews, creating photo collages, tweeting about specific topics etc. The same approach has been adopted within the MLEARN project at CSU university [8], [12] where different available apps were used for several purposes: iTunes $U$ for courses delivery, self-assessment tools and an ePortfolio App.

Finally, in the third category, new private mobile apps and tools are implemented to support particular learning tasks. In some specific contexts, no mobile Apps are available that meet accurately the objectives of the teacher's pedagogical scenario. In [13], a mobile tutoring system for contextual learning using QR-code labeling of objects in the real world has been developed. The QR codes are decoded by a mobile app providing educational support to the learner both in natural language and through the display of multimedia elements. In [14], QR codes are added to printed material and assignments, which allows students to view lesson-related materials (web links, video, etc.). Medical students in [15] used an augmented reality app (mARble) to practice medical diagnosis, predefined markers are placed on the body of other students by the tutor, corresponding to the wound patterns that the student is expected to explore. Then, they "examine" each other using the iPhone, they can add snapshots of the augmented image and create flashcards for memorization. Another example from [16] is TSOICHEM, a mobile app for "general chemistry" course, a dynamic alternative to classic flashcards, where students use the touch-screen feature to highlight the bonds and atoms that form a molecule.

\section{Classification of Educational Mobile Use}

\section{A. Previous Mobile-Learning Related Classifications}

For different purposes, many authors made different classifications related to several aspects of the mobile learning concept.

In [17], authors assigned mobile activities into six categories according to relevant learning theories: 1) Behaviorist activities, are activities that promote learning as a change in learners' observable actions (e.g. classroom response systems, formative assessment, peer feedback); 2) Constructivist activities are activities in which learners actively construct new ideas or concepts (e.g. participatory simulations); 3) Situated learning activities are those that promote learning within an authentic context and culture (e.g. context aware applications); iv)Collaborative activities are activities that promote learning through social interactions; v)Informal and life-long activities are those supporting learning outside a dedicated learning environment and formal curriculum; and finally, vi) learning and teaching support activities are activities that assist in the coordination of learners and resources for learning activities (e.g. personal organization and administrative support).

In [18], authors propose a framework for ubiquitous learning environments where they identify relevant features necessary to design an environment support of learning and competences development for mobile workers. We report this work here because the target learners are supposed to be learning in a mobile context. The framework includes the following functionalities: communication and collaboration, multimedia, online repository of documents, evaluation tools, location service, context management as well as reminders of activities and events.

In another work [19], authors tried to characterize m-learning according to the expected role of the mobility for learning. Six categories of m-learning have been defined: 1) Technology-driven mobile learning: when specific technological innovation is deployed in an academic setting to demonstrate technical and pedagogical feasibility; 2) Miniature but portable e-Learning : solutions already used in 'conventional' e-Learning and merely using mobile technologies as flexible replacements for static desktop technologies; 3) Connected classroom learning: technologies in classroom settings to support collaborative learning, 
sometimes connected to other classroom technologies such as interactive whiteboards; 4) Informal/personalized/situated mobile learning : when mobile technologies are enhanced with additional functionalities such as location-awareness, where learners can take a mobile device into an authentic context, or use it while moving around a context-aware environment in a specially equipped location such as museum; 5) Mobile training/performance support: when mobile technologies are used to improve the productivity and efficiency of mobile workers by delivering information and support just in-time for their immediate priorities; and 6) Remote/rural development mobile learning: when mobile technology is used to address environmental and infrastructural difficulties where 'conventional' e-learning technologies would fail.

In [20], another classification is given. It is related to the possible ways of integrating mobile learning into an overall institutional strategy while maintaining simplicity and ease of use. The author points out four simple options for m-learning implementation into a learning environment: 1) BYOD (Bring Your Own Device): the simplicity is related to the use of available learners' devices; 2) Mobile social media: making use of the existing tools of social networking, social bookmarking, multimedia sharing, collaborative Mind-mapping, etc.; 3) Mobile apps: including native apps and mobile web applications, whether they're publically available for download or developed for private institutional use; and 4) Mobile sensors: taking benefit from the natively available sensors and other components on any mobile device; Which makes them more context-aware of their environment and provides more opportunities for adapting learning media (e.g GPS, barcode sensors, accelerometer, etc.).

In [21], the purpose was to find free and simple mobile apps to be experimented in a higher education learning process. The authors presented the useful mobile tools for education in the following categories: 1) communication tools (e.g. social networking and instant messaging); 2) information sharing (e.g. cloud applications); 3) student organization (e.g. schedule management tools); 4) classroom tool (e. g note-taking, real-time surveys); 5) basic everyday tools (e.g. calculators, converters, translators, etc.); and 6) applications oriented to engineering (e.g. for some educational specialties).

\section{B. Synthesis}

The main purpose of the previously cited classifications was to facilitate the adoption of m-learning from different perspectives: as an institutional solution, in informal contexts or even in a more specific learning context. The used classification criteria were based either on pedagogical aspects related to mobility, or mobile activities that could be included in education.

Regarding the last point, the identified mobile learning activities either don't cover the totality of actions usually included in every learning process, or cover them in a very general manner. In fact, there was no mention of the use of the identified activities for some objectives as specific as construction and restitution of knowledge, evaluation, or self-evaluation for the regulation of one's own learning process.
Furthermore, there were no clear distinction between learners' and teachers' point of view. In other words, between mobile learning and mobile teaching activities. Also, some other particular aspects of learning haven't been mentioned, such as emotional and affective aspects, even though they're also affected by mobile technologies.

\section{Our Classification Criteria}

In order to address these deficiencies, we propose to make a classification of all mobile use categories for educational purposes, taking into account both learners' and teachers' visions.

For this purpose, we started by analyzing elementary learning actions that learners carry out during every learning process, in order to categorize them and also to position mobile learning activities accordingly. Two main concepts that we found appropriate for this analysis are Bloom's taxonomy and learning strategies paradigm, which both strongly focus on cognitive aspects of learning. Furthermore, we want to cover all learners' types, including university students and life-long learners, which have some particular aspects, since they are mostly adults, they are conscious of the learning process and are supposed to self-control, they are also more exposed to stress and demotivation. Therefore, we adopted learning strategies as a guiding theory, since it also puts the focus on affective and meta-cognitive aspects of learning.

We recall that, according to M. O’Malley [22], learning strategies are "the mental processes - special thoughts or behaviors - that individuals use to help them comprehend, learn, or retain new information". A. Boulet et al. [23] define four categories of learning strategies most pedagogy experts agree upon:

- Cognitive strategies: strategies which help students organize information, mentally and/or physically manipulate materials, and apply specific techniques to a learning task. C. Begin [24] defines ten cognitive strategies divided into two sub-categories:

Strategies for knowledge processing: "selection," "repetition," "decomposition," "comparison," "elaboration" and "organization"

Strategies for executing (reusing) knowledge: "evaluation," "verification," "production" and "transformation"

- Resource management strategies: Strategies allowing learners to adapt to their environment and make use of its resources for learning purposes. Three subcategories are defined related to time management, human resources and environmental resources management.

- Metacognitive strategies: those which help learners monitor their own comprehension and thinking in general. In other words, self-evaluation and monitoring for self-regulation of learning.

- Affective strategies: Affective strategies are learning strategies concerned with managing students' emotions and creating a positive affective environment in order to help their learning. The three defined subcategories are related to developing and maintaining motivation, for increasing concentration and for anxiety and stress control. Additionally, the teacher is a key actor in most mobile 
learning scenarios, particularly in formal contexts. His role could get more complicated when putting additional effort to manage mobile technologies, especially interactive and real time tools in class, leading him, in some cases, to a cognitive overload and a possible abandon of the concept altogether.

Thus, in our classification we also consider to define mobile uses support for the teacher, which along with mobile uses for learner support, constitute a practical guide for teachers when integrating or implementing a pedagogical m-learning scenario.

\section{OUR ClASSIFICATION}

\section{A. Overview}

We will present our classification according to three dimensions (Fig. 1):

- Learner's support, including three learning strategies sub-dimensions: cognition, resource management and metacognition.

- Teacher's support

- Affective support, for both teachers and learners, in terms of motivation, concentration and stress management.

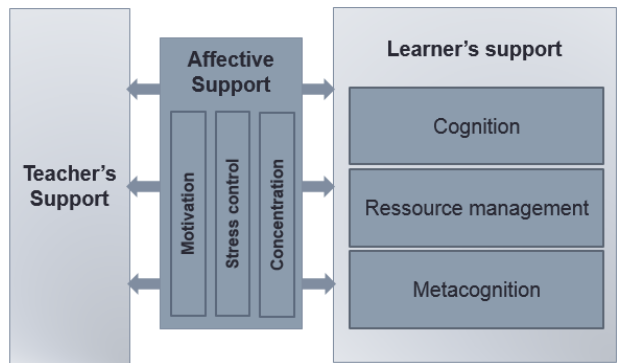

Fig. 1. Dimensions of our educational mobile use classification.

In order to identify the possible mobile use categories in each of these dimensions, and also to validate our classification, we did a literature review of previous experiences conducted in formal settings (cf. Parag. II. B.). Each of these studies had particular purposes for implementing m-learning, and therefore didn't need to include every existing educational mobile use in their scenarios.

Consequently, we also explored educational mobile apps in the two leading mobile app stores, in terms of numbers of apps and downloads: Google's Google play (for Android devices) with 1.6 million apps in 2015, and Apple's iTunes (for iOS devices) 1.5 million apps in 2015 [25].

Mobile apps are software applications designed to run on smartphones, tablet computers and other mobile devices, and usually written for a specific operating system. They are typically available through native distribution platforms called app stores or app marketplaces.

In Google Play and iTunes App Store, and every other app store, apps are put into different categories depending on their development purpose. We explored the top downloaded apps of all categories in order to validate our classification and identify the mobile use subcategories for learning and teaching. Most of the educational apps can be found in the "Education" category, however, a lot of apps that could be used for educational purposes can be found in other categories, such as "Books and references," "Productivity" and "Tools". In the following paragraphs, we describe some of the existing tools according to our classification.We start by introducing the classification for learners and then for teachers.

\section{B. Mobile Use for Learner's Cognitive Support}

In the cognitive learning process, we define three main actions (Fig. 2): knowledge construction and memorization (cognitive strategies for knowledge processing) and knowledge evaluation (cognitive strategies for executing knowledge).

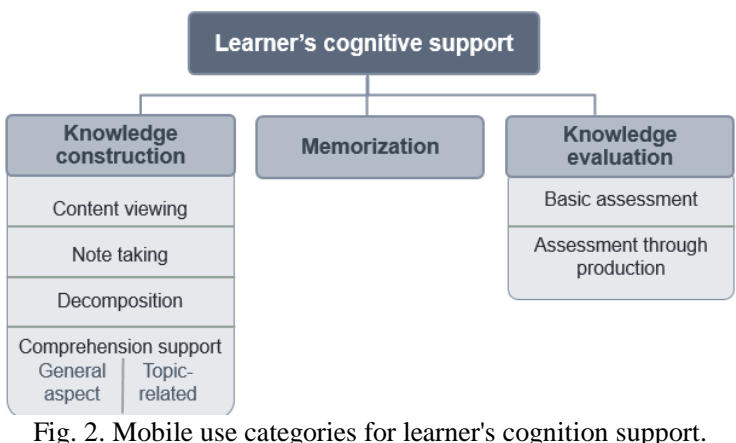

The corresponding mobile uses are as follows:

Knowledge construction: including mobile use for selecting, viewing and processing information. This could be achieved through:

- Content viewing: mobile devices with large screens are quite convenient for viewing content, e.g. reading eBooks can feel similar to holding paper books. It is also possible to listen to audio books and guides, which is helpful while traveling or driving. There are even specific types of content especially designed for touch-screen devices, like interactive eBooks and 3D guides, sometimes including animations and audio comments as well, which makes content viewing a more active experience for learners.

- Note taking: mobile makes it easy to take notes anytime without needing to carry around a pen and paper notebook, especially, using hand writing tools. Furthermore, it allows taking more rich notes in various formats: taking pictures, recording audio and video, tools for saving helpful web pages for later reading (Bookmarking) and even for documents scanning.

- Decomposition: passive viewing or listening to content is not enough for a good understanding. Currently existing mobile tools can help learners divide or formulate content for better personal understanding and sense-making. E.g. tools for annotating parts of text, for creating and organizing flashcards and Mind-maps for later revision, or for creating diagrams and layouts, which is easier than making and drawing them manually.

- Comprehension support: general aspect or topic-related support. By using some mobile tools as complementary help during some learning activities. For example, instead of constantly carrying a calculator, a dictionary, a grammar book etc.; their mobile alternatives are much easier to use: searching for a word in mobile dictionary is much easier than a paper dictionary, some dictionaries even include voice search, and some photo-calculator apps include 
equation recognition and resolution. In the subcategory of learning topic-related apps, it is relevant to apply appropriate learning theories and didactic solution. Also, various apps related to understanding specific subjects exist: e.g. electric simulators, programming IDE, educational games, etc. In Fig. 3 we give some statistics on "comprehension support" tools, which constitute $48 \%$ of the top 200 Apps of the category "Education" in Google Play.

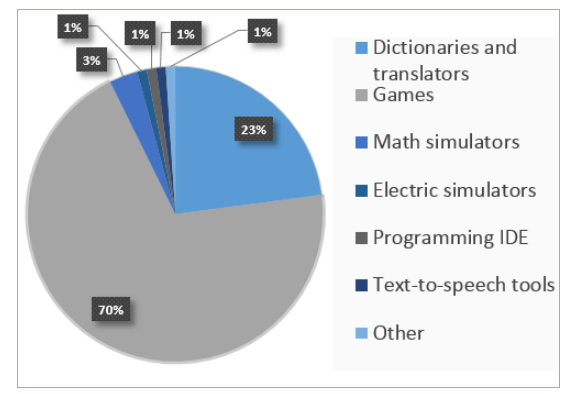

Fig. 3. Comprehension support tools.

Memorization: compared to classic ways, some mobile tools can be particularly adapted to memorizing and revising information, such as mind-maps, text-memorizers and flashcards. This latter can be used in a simple and intuitive way on mobile, containing pictures, sounds and animations.

Knowledge evaluation: using mobile to evaluate the learner's level of understanding which could be achieved through:

- Basic assessment: usual quizzes and exercises can be more appealing on mobile, using the touch screen (mostly as drag-and-drop exercises) and including sound effects and Gamification aspects (levels, high-scores, timing, etc.)

- Assessment through production: more elaborate evaluation requires the creation of content, different mobile tools exist for different assignment types: photo editing and collage, video montage and animations creation, text editors, writing tools which could include dictation and automatic conversion to text, art tools etc.

\section{Mobile Use for Learner's Resource Management Support}

In the resource management strategies, we define three corresponding mobile use categories (Fig. 4):

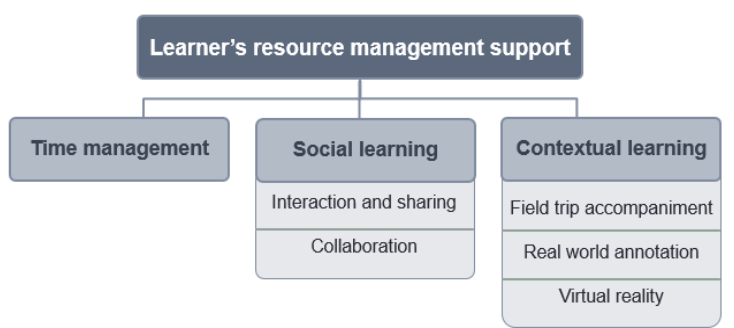

Fig. 4. Mobile use categories for learner's resource management support.

Time management: what makes mobile especially useful for time management are notifications and reminders to users. A lot of mobile apps exist for customized calendars, creating schedules, managing deadlines, to-do lists etc.

Social learning: mobile also facilitates taking advantage of human resources, in other words learning with and from others through two types of use:
- Interaction and sharing: in order to interact with peers or experts, to share useful content or personal creations, mobile allows instant messaging, sharing and feedback using chat and social networking tools.

- Collaboration: various shared work spaces are also available on mobile, such as collaborative note-taking, collaborative file editing, real-time surveys, shared virtual white boards (real-time writing, drawing, etc.).

Contextual learning: mobile devices, considered as ubiquitous devices, represent the perfect tool to exploit surrounding environmental resources. For education purposes, this could be achieved through three main uses:

- Field trip accompaniment: using mobile tools to capture information from the real world (e.g. pictures and video recording) or as accompanying guides during educational visits.

- Real world annotation: interacting with the surrounding environment by adding QR-codes or other special markers to real objects. As response, mobile shows different types of content: opening web pages, videos etc.

- Virtual reality: virtual visits to museums, parks, historic sites or imaginary worlds.

\section{Mobile Use for Learner's Metacognitive Support}

Metacognitive activities are related to self-monitoring and auto-directing. We named the corresponding mobile use as "Self-monitoring/regulation" which is usually related to time management and evaluation, but more focused on the knowledge itself and individual's level of performance. Several available mobile tools allow setting learning goals and monitoring grades, measuring performance levels, based on revision time per subject for example.

The following figure (Fig. 5) represents the statistics of learner's supporting tools among the top 200 Apps of the category "Education" in iTunes App store.

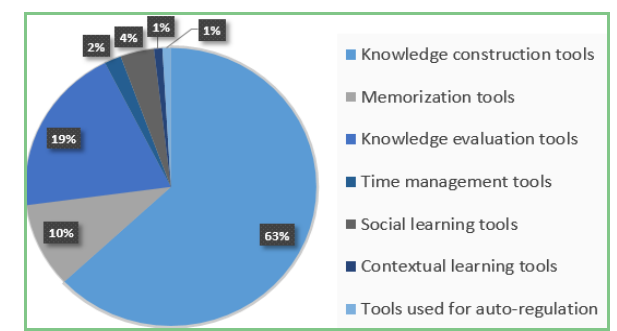

Fig. 5. Learner's support tools according to our classification.

\section{E. Mobile Use for Teacher's Support}

As assistance to teachers, we identified four mobile uses (Fig. 6):

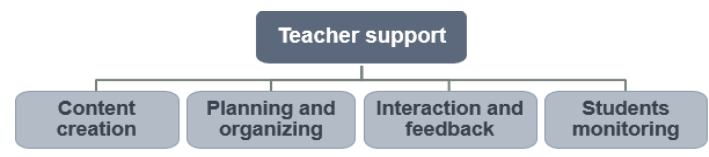

Fig. 6. Mobile use categories for teacher's support.

Content creation: in addition to classical tools for content creation like text editors, spreadsheet etc., mobile devices (tablets in particular) can make it easier to write, draw, make video captures of handwritten explanation, make audio and video recordings, or even create interactive eBooks using drag-and-drop features and integrate it into the content to be 
delivered to the learners.

Planning and organizing: several existing tools for time management are aimed at organizing the different tasks related to teaching: course planning, exam schedules, meeting notifications etc.

Interaction and feedback: several existing mobile tools and platforms allow teachers interacting with peers, with students (and in some cases with parents), using, interchangeably various interaction tools as messaging, notification, SMS etc.

Students monitoring: a lot of mobile tools allow teachers to perform an individual monitoring of learning, outcomes and students behavior. As example, we can cite: attendance managers, homework follow-up, grading apps, in-class device monitoring (e.g. for blocking internet access) etc.

In Fig. 7, we give some statistics on "teacher's support" tools, from the app collection "Tools for teachers", in the "Education" category in iTunes App store.

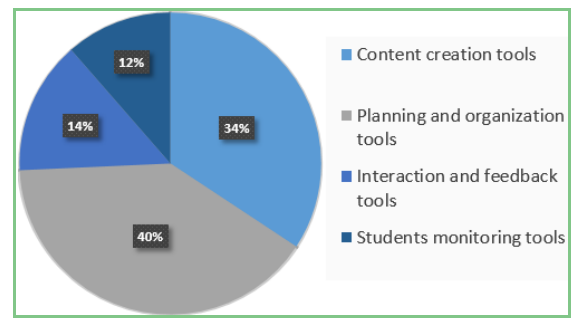

Fig. 7. Teacher's support tools according to our classification.

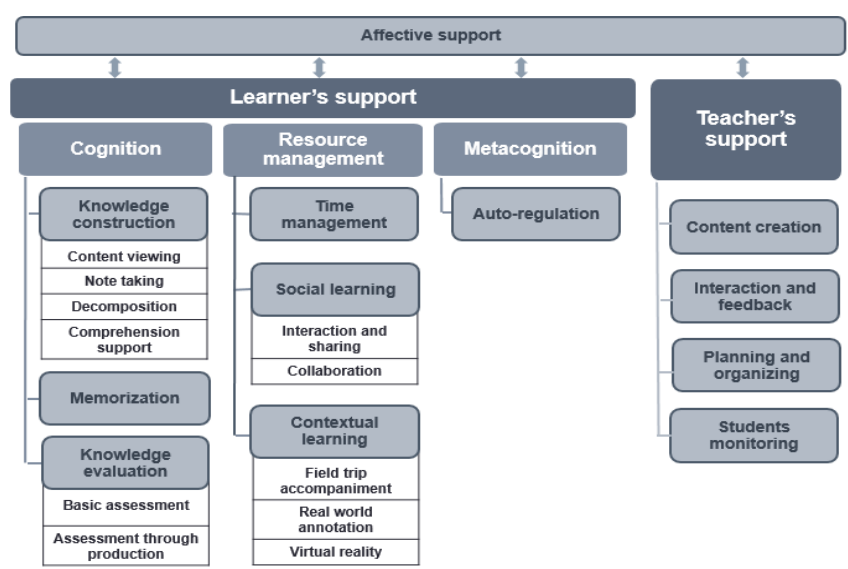

Fig. 8. Diagram of elementary educational mobile use of our classification.

\section{F. Mobile Use for Affective Support}

The three affective aspects of learning in terms of concentration, motivation and stress control are unarguablly important in every learning context. We believe that these three aspects should be taken into consideration while designing mobile learning tools in relation with all the previous categories, both for learners and teachers. The concentration aspect is more relevant for learners, while motivation and stress control can be of great help for both.

For motivation, a rewarding feature added to quiz tools for example, will probably increase the learner's motivation (e.g high scores and badges). Whereas the technical quality of the mobile tool as well as the fluidity of its ineterface and its ease of use could be factors of motivation for learners and teachers.

For concentration, some time management features issued from the meta-cognitive tools can help increasing concentration and overcoming the escape of mind, such as notifications and reminders of revision time or deadlines.

For stress control, the animated and fun aspect of some mobile tools like interactive eBooks, virtual visits and animated flashcards could considerably reduce the stress of learning compared to a classical context. Furthermore, some existing tools such as anti-anxiety and relaxation apps could be benificial for both learners and teachers. We propose that, in a m-learning environment, the user should have periodical access, limited in time, to these tools, which permits him to take a break, to relax and continue learning (learner) or working (teacher) through the environment with energy.

As a summary, we present in Fig. 8 recap of all the identified mobile uses according to the three dimensions of our classification.

\section{CONCLUSION}

In this paper, we aimed at providing educators and learning designers with a generic tool that helps them designing a functionnaly complete mobile learning environment. Therefore, we proposed a classification of mobile educational uses based on learning strategies, and taking into account both learners' and teachers' activities. We are currently designing a social mobile environment, support of a community of pratice of learners in the frame of a university scientific learning module.

\section{REFERENCES}

[1] S. Bidin and A. A. Ziden, "Adoption and application of mobile learning in the education industry," Procedia - Social and Behavioral Sciences, 2013, vol. 90, pp.720-729

[2] J. Colley and G. Stead, "Take a bite: Producing accessible learning materials for mobile devices," Learning with Mobile Devices: Research and Development, London: Learning and Skills Development Agency, 2003.

[3] A. Bokma, C. Bowerman, and P. Graham, "Adaptive navigation for mobile devices," Learning with Mobile Devices — Research and Development, LSDA Publishing, 2004, pp. 61-67.

[4] A. Fernàndez and M. C. Mayorga, "Learning, tools for java enabled phones: An application to actuarial studies," in Proc. the International Conference.

[5] C. O'Malley, G. Vavoula et al., Guidelines for Learning/Teaching/Tutoring in a Mobile Environment, 2003.

[6] J. Traxler, "Defining, discussing, and evaluating mobile learning," International Review of Research in Open and Distance Learning, 2007, vol. 8, no. 2 .

[7] R. Picek and M. Grcic "Evaluation of the potential use of mlearning in higher education," in Proc. the ITI $201335^{\text {th }}$ In. Conf. on Information Technology Interfaces, Junes 24-27, Croatia, 2013.

[8] P. Uys. (2012). Creating and executing a successful mobile learning strategy - A Charles Sturt University case study. [Online]. Available: http://fr.slideshare.net/puys/creating-and-executing-a-successful-mobi le-learning-strategy-a-charles-sturt-university-case-study

[9] N. Galanis, M. J. Casany, M. Alier, and E Mayol, "Building a community: The moodbile perspective," presented at IEEE 38th Annual International Computers, Software and Applications Conference Workshops, 2014

[10] Official website of the blackboard LMS. [Online]. Available: http://www.blackboard.com/

[11] L. Ducate and L. Lomicka, "Going mobile: Language learning with an iPod touch in intermediate French and German classes," Foreign Language Annals, vol. 46, issue 3, pp. 445-468, 2013.

[12] J. Brann, P. Uys, L. Griffin, T. Klapdor, S. Thomson, R. Fell, M. Morton-Allen, and M. Harris, Mobile Learning Initiative Investigation Report, 2011.

[13] O. D. Pietro, G. Frontera, and P. Bucci, "Mobile tutoring for situated learning and collaborative learning in AIML application using QR-Code," presented at Sixth International Conference on Complex, Intelligent, and Software Intensive Systems, 2012. 
[14] M. F. Tretinjak, "The implementation of QR codes in the educational process," MIPRO 2015, May 2015, Opatija, Croatia.

[15] U. V. Albrecht, M. Behrends, H. K. Matthies, and U. V. Jan, "Medical students experience the mobile augmented reality blended learning environment (Marble (): An attractive concept for the net generation?" Ubiquitous and Mobile Learning in the Digital Age, 2013.

[16] M. Y. Tsoi and S. Dekhane, "TsoiChem: A mobile application to facilitate student learning in organic chemistry," presented at 11th IEEE International Conference on Advanced Learning Technologies, 2011.

[17] L. Naismith, M. Sharples, G. Vavoula, and P. Lonsdale, "Literature review in mobile technologies and learning," A NESTA Futurelab Series Report 11, 2004.

[18] A. Zanela, M. Kich, and E. Schlemmer, "A framework for the design of ubiquitous learning applications," in Proc. the 42nd Hawaii International Conference on System Sciences, 0 Jan. 2009.

[19] J. M. Traxler, "Learning in a mobile age," International Journal of Mobile and Blended Learning, vol. 1, no. 1, January-March 2009.

[20] I. I. Waard, "Using BYOD, mobile social media, apps, and sensors for meaningful mobile learning," Increasing Access through Mobile Learning, 2014.

[21] J. S. Artal-Sevil, J. L. Bernal-Agustín, and J. A. Domínguez, "M-Learning (mobile learning) in higher education. The impact of smartphone as interactive learning tool," in Proc. EDULEARN15 Conference, Spain, pp. 8212-8221.

[22] J. M. O'Malley and A. U. Chamot, Learning Strategies in Second Language Acquisition, 1990.

[23] A. Boulet, L. Savoie-Zajc, and J. Chevrier, Learning Strategies at University, 1996

[24] C. Bégin,"Learning strategies: A simplified framework," Revue Des Sciences de l'Education, vol. 34, no. 1, 2008, pp. 47-67.

[25] Statistics and facts about App stores. [Online]. Available: http://www.statista.com/topics/1729/app-stores/

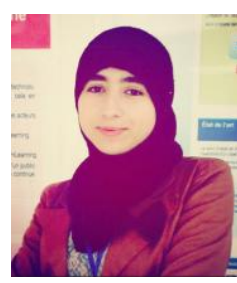

Oumayma Chergui received her master's degree in intelligent systems and networks from the Faculty of Science and Technology, in University Sidi Mphamed Ben Abdellah, Fez, Morocco, in 2013. She's currently a Ph.D student at the intelligent systems and applications laboratory at the Faculty of Science and Technology, Fez. Her research interests include mobile learning, social learning, learning platforms, and ICT in higher education. Ms. Chergui is a member of the IEEE Morocco Section.

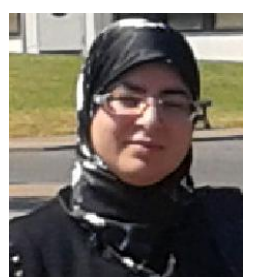

Ahlame Begdouri is an HDR professor of computer science in the Faculty of Science and Technology of Fez-University of Sidi Mohamed Ben Abdellah. She is interested in mobile learning since 2008, her current research interests concern mobile/social learning and context aware adaptation. Prof. Begdouri is the head of the VIA research group of the SIA Lab (Syntème Intelligents et Applications), she was the coordinator of an FP7 project called MoICT (2011-2014), member of IEEE Moroccan Education Chapter, program committee chair of several international conferences (WNGN'08, NGNS'09, CIST'11, CIST'12, ELED'16 workshop).

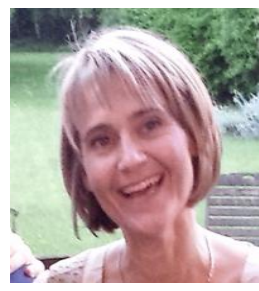

Dominique Leclet-Groux is an associate professor and HDR (French Acronym: Habilité à Diriger des Recherches) at the MIS laboratory, Université de Picardie Jules Verne, Amiens, France. She is interested in context awareness and adaptation in a mobile environment community computer but also virtual environments and case-based reasoning. 